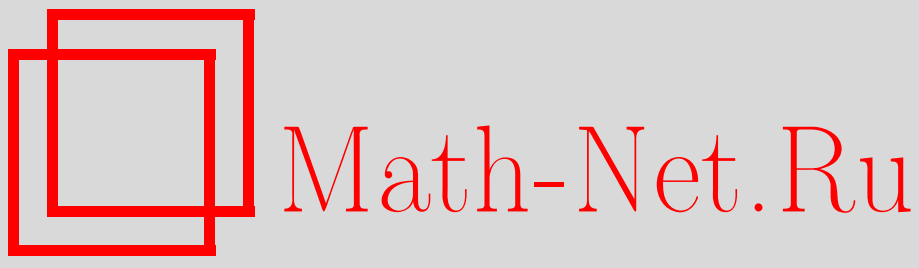

О. И. Богоявленский, Общие алгебраические тождества для тензоров Нийенхейса и Хаантжеса, Изв. РАН. Сер. матем., 2004, том 68, выпуск 6, 71-84

DOI: https://doi.org/10.4213/im511

Использование Общероссийского математического портала Math-Net.Ru подразумевает, что вы прочитали и согласны с пользовательским соглашением

http: //www. mathnet.ru/rus/agreement

Параметры загрузки:

IP: 54.224 .60 .19

26 апреля 2023 г., 18:16:47 
УДК $514.7+514.8$

\author{
О.И. Богоявленский
}

\title{
Общие алгебраические тождества для тензоров Нийенхейса и Хаантжеса
}

\begin{abstract}
Получены общие алгебраические тождества для тензоров Нийенхейса и Хаантжеса на произвольном многообразии $M^{n}$. Для $n=3$ выведены специальные алгебраические тождества, связанные с формой Картана-Киллинга $(u, v)_{H}$.

Библиография: 22 наименования.
\end{abstract}

\section{§1. Введение}

1.1. В известных работах [1], [2] А. Нийенхейс сформулировал задачу о взаимоотношениях между тензорами $N_{B(A)}(u, v)$ и $N_{A}(u, v)$, где $(1,1)$-тензор $B(A)$ является произвольным многочленом от $(1,1)$-тензора $A$ на некотором $n$-мерном многообразии $M^{n}$. В настоящей работе дается полное решение задачи Нийенхейса и решение более общей задачи о взаимоотношениях между тензорами Нийенхейса $N_{B, C}(u, v)$ и $N_{A}(u, v)$, где $B$ и $C$ - произвольные многочлены от $A$. В $\S 3$ представлены явные формулы, связываюшие тензоры $N_{B(A)}(u, v), N_{B, C}(u, v)$ и $N_{A}(u, v)$. Для вывода этих формул мы вводим в $\S 2$ представление коммутативного кольца $R[z, \lambda, \mu]$ многочленов от трех независимых переменных в пространстве $(1,2)$-тензоров на многообразии $M^{n}$.

Мы выводим явные формулы, связывающие тензоры Хаантжеса $H_{B(A)}(u, v)$ и $H_{A}(u, v)$. Одно из этих тождеств доказывает, что тензор Хаантжеса $H_{A}(u, v)[3]$ является калибровочным инвариантом по отношению к калибровочным преобразованиям $(1,1)$-тензора $A_{j}^{i}(x)$, в отличие от тензора Нийенхейса $N_{A}(u, v)$.

В $\S 4$ доказано некоторое общее алгебраическое тождество для тензора Нийенхейса, которое связано с характеристическим многочленом $(1,1)$-тензора $A_{j}^{i}(x)$. Получено обшее алгебраическое тождество для тензора Хаантжеса $H_{A}(u, v)$.

Альтернированный $(1,2)$-тензор Нийенхейса $N_{A}(u, v)$ и тензор Хаантжеса $H_{A}(u, v)$ определяют деформацию структур неассоциативных и альтернированных алгебр в касательном расслоении $T\left(M^{n}\right)$. В работах [4]-[6] мы ввели $(1,3)$-тензоры $B_{N j k \ell}^{i}$ и $B_{H j k \ell}^{i}$, которые характеризуют отклонение этих алгебраических структур от алгебр Ли. Обшая теория деформаций алгебр Ли развита в рабо$\operatorname{Tax}[7],[8]$.

В $\S 5$ показано, что произвольный $(1,1)$-тензор $A_{j}^{i}(x)$ на трехмерном многообразии $M^{3}$ является симметрическим по отношению к билинейной форме $(u, v)_{H}$, связанной с тензором Хаантжеса $H_{A}(u, v)$. Доказано, что тензор Хаантжеса $H_{A}(u, v)$ определяет деформацию структур алгебр Ли в касательном расслоении $T\left(M^{3}\right)$, и выведены алгебраические тождества с использованием формы Картана-Киллинга $(u, v)_{H}$. 
1.2. Тензор Нийенхейса определяется формулой [1]

$$
N_{A}(u, v)=A^{2}[\tilde{u}, \tilde{v}]+[A \tilde{u}, A \tilde{v}]-A([A \tilde{u}, \tilde{v}]+[\tilde{u}, A \tilde{v}]),
$$

где $u, v$-касательные векторы в точке $x \in M^{n}$ и $\tilde{u}, \tilde{v}$ - произвольные векторные поля, продолжающие векторы $u, v$ (выражение (1.1) не зависит от продолжений $\tilde{u}$ и $\tilde{v})$.

Тензор Нийенхейса используется во многих задачах дифференциальной геометрии и математической физики; однако до настояшего времени основные применения тензоров Нийенхейса и Хаантжеса [3] были связаны с условиями их тривиальности: $N_{A}(u, v)=0$ или $H_{A}(u, v)=0$. Теорема Ньюландера-Ниренберга [9] утверждает, что почти комплексная структура $A(x), A^{2}(x)=-1$, является комплексной тогда и только тогда, когда тензор Нийенхейса $N_{A}(u, v)$ тривиален. Из теоремы Нийенхейса-Хаантжеса [1], [3] следует, что для $(1,1)$-тензора $A_{j}^{i}(x)$ с действительными и неравными собственными значениями на гладком многообразии $M^{n}$ линейные комбинации любых $n-1$ собственных векторов порождают интегрируемое распределение, если и только если тензор Хаантжеса $H_{A}(u, v)$ тривиален.

Условие $N_{A}(u, v)=0$ используется в работах [10], [11] в качестве достаточного условия для существования специальных структур алгебр Ли в пространстве векторных полей на многообразии $M^{n}$. Оно применяется в [12] как условие интегрируемости $G$-структур на $M^{n}$. Теорема Гельфанда-Дорфман-Магри-Мороси [13], [14] утверждает, что две пуассоновы структуры $P_{1}$ и $P_{2}$ являются совместимыми в смысле Магри [15] тогда и только тогда, когда $N_{A}(u, v)=0$, где $A=P_{1} P_{2}^{-1}$.

Условие $N_{A}(u, v)=0$ используется в качестве определения структур Пуассона-Нийенхейса (см. [14], [16], [17]) и как определение $G$-многообразий Нийенхейса (см. [18]), а также применяется в [19], [20] как достаточное условие существования законов сохранения для систем уравнений в частных производных

$$
u_{t}^{i}=\sum_{j=1}^{n} A_{j}^{i}\left(u^{1}, \ldots, u^{n}\right) u_{y}^{j}, \quad i=1, \ldots, n .
$$

В работах [4]-[6] были применены ненулевые тензоры Нийенхейса и Хаантжеса при изучении необходимых условий существования гамильтоновых и бигамильтоновых структур [21] для систем (1.2). В статье [22] использованы ненулевые тензоры Нийенхейса и Хаантжеса при исследовании необходимых условий существования законов сохранения для систем (1.2).

\section{§2. Представление кольца многочленов $R[z, \lambda, \mu]$ в пространстве $(\mathbf{1}, \mathbf{2})$-тензоров}

2.1. Рассмотрим коммутативное кольцо $R_{3}=R[z, \lambda, \mu]$ многочленов от трех независимых переменных $z, \lambda, \mu$ с коэффициентами, зависящими от точки $x$ многообразия $M^{n}$. Элементами кольца $R_{3}$ являются многочлены

$$
S(z, \lambda, \mu)=\sum_{i, j, k=1}^{N} a_{i j k}(x) z^{i} \lambda^{j} \mu^{k}, \quad x \in M^{n},
$$

где коэффищиенты $a_{i j k}(x)$ являются произвольными гладкими функциями на $M^{n}$. 
Определим представление $T$ кольца $R_{3}$ в линейном пространстве $(1,2)$-тензоров $V(u, v)$. Это представление зависит от произвольного $(1,1)$-тензора $A(x)$ и определено для произвольного многочлена $S(z, \lambda, \mu)($ см. $(2.1))$ формулой

$$
\left(T_{S} V\right)(u, v)=\sum_{i, j, k=1}^{N} a_{i j k}(x) A^{i} V\left(A^{j} u, A^{k} v\right) .
$$

Представление (2.2) обладает стандартными свойствами:

$$
T_{S_{1}+S_{2}}=T_{S_{1}}+T_{S_{2}}, \quad T_{S_{1} \cdot S_{2}}=T_{S_{2} \cdot S_{1}}=T_{S_{1}} \cdot T_{S_{2}} .
$$

Первое тождество в (2.3) очевидно. Для доказательства второго тождества в (2.3) предположим сначала, что оператор $A(x)$ имеет неравные собственные значения $\lambda_{1}(x), \ldots, \lambda_{n}(x)$ и базис собственных векторов $e_{1}(x), \ldots, e_{n}(x)$. Очевидно, мы имеем

$$
T_{S} V\left(e_{i}, e_{j}\right)=S\left(A, \lambda_{i}, \lambda_{j}\right) V\left(e_{i}, e_{j}\right) .
$$

Отсюда для произвольных многочленов $S_{1}$ и $S_{2}$ следует равенство

$$
T_{S_{1} \cdot S_{2}} V\left(e_{i}, e_{j}\right)=T_{S_{1}}\left(T_{S_{2}} V\left(e_{i}, e_{j}\right)\right)=T_{S_{2} \cdot S_{1}} V\left(e_{i}, e_{j}\right) .
$$

Равенство $(2.3)$ следует из $(2.5)$ в силу билинейности тензоров $V(u, v)$. Для оператора $A\left(x_{1}\right)$, имеющего совпадающие собственные значения, мы рассмотрим некоторое его непрерывное возмущение $\tilde{A}(x)$, которое имеет различные собственные значения вне точки $x_{1}$. Тогда тождество $(2.3)$ для оператора $A\left(x_{1}\right)$ следует по непрерывности из невырожденного случая, рассмотренного выше.

2.2. Для любых коммутируюших $(1,1)$-тензоров $B_{j}^{i}(x)$ и $C_{\ell}^{k}(x)$ Нийенхейс определил в работе [1] $(1,2)$-тензор

$$
N_{B, C}(u, v)=B C[\tilde{u}, \tilde{v}]+[B \tilde{u}, C \tilde{v}]-B[\tilde{u}, C \tilde{v}]-C[B \tilde{u}, \tilde{v}] .
$$

Тензор Нийенхейса (1.1) является специальным случаем тензора (2.6) для $B=$ $C=A$. Тензор $N_{B, C}(2.6)$ имеет следуюшие компоненты:

$$
\left(N_{B, C}\right)_{j k}^{i}=B_{j}^{\alpha} C_{k, \alpha}^{i}-C_{k}^{\alpha} B_{j, \alpha}^{i}+C_{\alpha}^{i} B_{j, k}^{\alpha}-B_{\alpha}^{i} C_{k, j}^{\alpha},
$$

где $B_{j, \alpha}^{i}=\partial B_{j}^{i}(x) / \partial x^{\alpha}$, и т. д.

Тензор Хаантжеса [3] определяется формулой

$$
H_{A}(u, v)=A^{2} N_{A}(u, v)+N_{A}(A u, A v)-A\left(N_{A}(A u, v)+N_{A}(u, A v)\right) .
$$

$\mathrm{B}$ терминах представления $T_{S}$ (см. (2.2)) формула (2.7) принимает вид

$$
H_{A}(u, v)=T_{D} N_{A}(u, v),
$$

где $D$ является многочленом:

$$
D(z, \lambda, \mu)=(z-\lambda)(z-\mu) .
$$

Очевидно, что любой $(1,2)$-тензор $W(u, v)$ вида

$$
W(u, v)=\sum_{m=0}^{N}\left(u\left(g_{m}\right) A^{m} v+v\left(h_{m}\right) A^{m} u\right)
$$

принадлежит ядру оператора $T_{D}$ :

$$
T_{D} W(u, v)=0 .
$$


2.3. Пусть $B(z)$ - некоторый многочлен, $B(z)=\sum_{m=0}^{k} b_{m}(x) z^{m}$. Будем многократно использовать известное тождество Безу

$$
B(z)-B(\lambda)=(z-\lambda) Q_{B}(z, \lambda),
$$

где

$$
Q_{B}(z, \lambda)=\sum_{m=1}^{k} b_{m}(x) \sum_{p+q=m-1} z^{p} \lambda^{q},
$$

и его следствие

$$
Q_{B}(z, \lambda)-Q_{B}(z, \mu)=(\lambda-\mu) R_{B}(z, \lambda, \mu),
$$

где

$$
R_{B}(z, \lambda, \mu)=\sum_{m=2}^{k} b_{m}(x) \sum_{p+q+r=m-2} z^{p} \lambda^{q} \mu^{r} .
$$

Тождество (2.13) имеет эквивалентный симметрический вид:

$$
\begin{gathered}
(\lambda-\mu) B(z)+(\mu-z) B(\lambda)+(z-\lambda) B(\mu) \\
=(\lambda-\mu)(z-\lambda)(z-\mu) R_{B}(z, \lambda, \mu) .
\end{gathered}
$$

Очевидно, что многочлены $Q_{B}(z, \lambda)$ и $R_{B}(z, \lambda, \mu)$ являются симметрическими по отношению к их аргументам.

2.4. Введем следуюшие $(1,2)$-тензоры:

$$
\begin{gathered}
\widetilde{X}_{B}(u, v)=\sum_{m=1}^{k} b_{m}(x) \sum_{p+q=m-1} A^{p} H_{A}\left(A^{q} u, v\right), \\
\widetilde{Y}_{B}(u, v)=\sum_{m=1}^{k} b_{m}(x) \sum_{p+q=m-1} H_{A}\left(A^{p} u, A^{q} v\right), \\
\widetilde{Z}_{B}(u, v)=\sum_{m=2}^{k} b_{m}(x) \sum_{p+q+r=m-2} A^{p} H_{A}\left(A^{q} u, A^{r} v\right) .
\end{gathered}
$$

Формулы $(2.2),(2.12)$ и (2.14) приводят к выражениям

$$
\widetilde{X}_{B}=T_{Q_{B}(z, \lambda)} H_{A}, \quad \widetilde{Y}_{B}=T_{Q_{B}(\lambda, \mu)} H_{A}, \quad \widetilde{Z}_{B}=T_{R_{B}(z, \lambda, \mu)} H_{A} .
$$

Из тождества (2.13) имеем

$$
T_{Q_{B}(z, \lambda)} H_{A}-T_{Q_{B}(z, \mu)} H_{A}=T_{\lambda-\mu} T_{R_{B}(z, \lambda, \mu)} H_{A},
$$

что вследствие (2.17) означает

$$
\widetilde{X}_{B}(u, v)+\widetilde{X}_{B}(v, u)=\widetilde{Z}_{B}(A u, v)-\widetilde{Z}_{B}(u, A v) .
$$

Алгебраическое тождество

$$
Q_{B}(\lambda, z)-Q_{B}(\lambda, \mu)=(z-\mu) R_{B}(z, \lambda, \mu)
$$

аналогично приводит к тензорному тождеству

$$
\widetilde{X}_{B}(u, v)-\widetilde{Y}_{B}(u, v)=A \widetilde{Z}_{B}(u, v)-\widetilde{Z}_{B}(u, A v) .
$$


ПрЕДЛОЖЕНИЕ 2.1. Если оператор $A\left(x_{1}\right)$ удовлетворяет алгебраическому уравнению

$$
B\left(A, x_{1}\right) \stackrel{\text { def }}{=} \sum_{m=0}^{k} b_{m}\left(x_{1}\right) A^{m}\left(x_{1}\right)=0,
$$

то в точке $x_{1}$ справедливы следующие тензорные уравнения:

$$
\begin{gathered}
\widetilde{X}_{B}(u, v)=0, \\
\widetilde{Y}_{B}(A u, v)=\widetilde{Y}_{B}(u, A v), \\
\widetilde{Z}_{B}(A u, v)=\widetilde{Z}_{B}(u, A v) .
\end{gathered}
$$

ДоКАЗАТЕЛЬСТво. Формулы $(2.17),(2.8),(2.9)$ и тождество Безу (2.11) влекут

$$
\widetilde{X}_{B}=T_{Q_{B}(z, \lambda)} T_{D} N_{A}=T_{z-\mu} T_{B(z)-B(\lambda)} N_{A}
$$

Отсюда мы получаем

$$
\widetilde{X}_{B}(u, v)=T_{z-\mu}\left(B(A) N_{A}(u, v)-N_{A}(B(A) u, v)\right)=0
$$

вследствие уравнения (2.20). Уравнение (2.23) следует из (2.18) и (2.21). Уравнение $(2.22)$ вытекает из $(2.19),(2.21)$ и (2.23).

ЗАмЕчАнИЕ 2.1. Пусть некоторьй $(k, l)$-тензор $U(A)$ аналитически зависит от компонент тензора $A_{j}^{i}(x)$ и их частных производных до конечного порядка $N$. Если тензор $U(A)$ равен нулю для всех $(1,1)$-тензоров $A_{j}^{i}(x)$ с неравными (комплексными) собственными значениями, то $U(A) \equiv 0$ для любого $(1,1)$-тензора $A_{j}^{i}(x)$. Это очевидно следует по непрерывности из невырожденного случая $A_{j}^{i}(x)$ с неравными собственньми значениями.

ЗАмЕчАниЕ 2.2 . Все рассматриваемые в настояшей работе тензоры $U(A)$ имеют вид

$$
U\left(A_{j}^{i}, \frac{\partial^{k} A_{j}^{i}(x)}{\partial x_{\alpha_{1}} \ldots \partial x_{\alpha_{k}}}\right)
$$

и являются многочленами по отношению к их аргументам. Поэтому тензоры $U(A)$ могут быть продолжены на комплексификацию касательного расслоения $T\left(M^{n}\right)$ и кокасательного расслоения $T^{*}\left(M^{n}\right)$. Мы будем иметь в виду это продолжение, когда $(1,1)$-тензор $A_{j}^{i}(x)$ имеет комплексные собственные значения и собственные векторы.

\section{§3. Алгебраические соотношения между тензорами Нийенхейса}

В этом параграфе мы выводим формулу, связываюшую тензор Нийенхейса $N_{B, C}(2.6)$ с тензором Нийенхейса $N_{A}(1.1)$. Эта формула дает полное решение задачи о взаимосвязи между тензорами $N_{B(A)}(u, v)$ и $N_{A}(u, v)$, поставленной Нийенхейсом в работах [1], [2]. 
Пусть $B(A, x)$ и $C(A, x)$ - произвольные многочлены с переменными коэффициентами:

$$
B(A, x)=\sum_{m=0}^{k} b_{m}(x) A^{m}(x), \quad C(A, x)=\sum_{m=0}^{k} c_{m}(x) A^{m}(x)
$$

Пусть $\lambda_{1}(x), \ldots, \lambda_{n}(x)$ - собственные значения оператора $A(x)$, соответствуюшие собственным векторам $e_{1}(x), \ldots, e_{n}(x)$. Операторы $B(A, x)$ и $C(A, x)$ имеют те же собственные векторы с собственными значениями $B\left(\lambda_{i}(x), x\right)$ и $C\left(\lambda_{i}(x), x\right)$.

Лемма 3.1. Для (1,2)-тензора $N_{B, C}(u, v)$ (2.6) имеет место формула

$$
\begin{aligned}
& N_{B, C}\left(e_{i}, e_{j}\right)=\left(B(A)-B\left(\lambda_{i}\right)\right)\left(C(A)-C\left(\lambda_{j}\right)\right)\left[e_{i}, e_{j}\right] \\
& \quad+\left(B\left(\lambda_{i}\right)-B\left(\lambda_{j}\right)\right) e_{i}\left(C\left(\lambda_{j}\right)\right) e_{j}+\left(C\left(\lambda_{i}\right)-C\left(\lambda_{j}\right)\right) e_{j}\left(B\left(\lambda_{i}\right)\right) e_{i} .
\end{aligned}
$$

Действительно, формула (3.2) следует из определения (2.6) прямым вычислением.

Teорема 3.1. Тензор $N_{B, C}(u, v)$ выражается через тензор Нийенхейса $N_{A}(u, v)$ с помощью формульи

$$
\begin{aligned}
N_{B, C}(u, v)= & \sum_{m, l=1}^{k} b_{m} c_{l} \sum_{p<m, q<l} A^{m+l-p-q-2} N_{A}\left(A^{p} u, A^{q} v\right) \\
& +\sum_{m=0}^{k}\left[B(A) u\left(c_{m}\right) A^{m} v-C(A) v\left(b_{m}\right) A^{m} u\right. \\
& \left.-u\left(c_{m}\right) B(A) A^{m} v+v\left(b_{m}\right) C(A) A^{m} u\right]
\end{aligned}
$$

ДоКАЗАТЕЛЬСТво. Предположим сначала, что оператор $A(x)$ имеет неравные собственные значения $\lambda_{1}(x), \ldots, \lambda_{n}(x)$. Используя тождества Безу

$$
B(z)-B(\lambda)=(z-\lambda) Q_{B}(z, \lambda), \quad C(z)-C(\lambda)=(z-\lambda) Q_{C}(z, \lambda),
$$

мы получаем

$$
\begin{gathered}
Q_{B}(\lambda, \lambda)=\frac{\partial B(\lambda)}{\partial \lambda}, \quad Q_{C}(\lambda, \lambda)=\frac{\partial C(\lambda)}{\partial \lambda}, \\
e(B(\lambda))=\sum_{m=0}^{k}\left(b_{m} e\left(\lambda^{m}\right)+e\left(b_{m}\right) \lambda^{m}\right)=Q_{B}(\lambda, \lambda) e(\lambda)+\sum_{m=0}^{k} e\left(b_{m}\right) \lambda^{m}, \\
e(C(\lambda))=\sum_{m=0}^{k}\left(c_{m} e\left(\lambda^{m}\right)+e\left(c_{m}\right) \lambda^{m}\right)=Q_{C}(\lambda, \lambda) e(\lambda)+\sum_{m=0}^{k} e\left(c_{m}\right) \lambda^{m},
\end{gathered}
$$


где $e$ - произвольньй касательньй вектор, $e \in T_{x}\left(M^{n}\right)$. Вследствие тождеств (3.4) и (3.5) формула (3.2) принимает вид

$$
\begin{aligned}
N_{B, C}\left(e_{i}, e_{j}\right)= & Q_{B}\left(A, \lambda_{i}\right) Q_{C}\left(A, \lambda_{j}\right)\left(A-\lambda_{i}\right)\left(A-\lambda_{j}\right)\left[e_{i}, e_{j}\right] \\
& +Q_{B}\left(\lambda_{i}, \lambda_{j}\right) Q_{C}\left(\lambda_{j}, \lambda_{j}\right)\left(\lambda_{i}-\lambda_{j}\right) e_{i}\left(\lambda_{j}\right) e_{j} \\
& +Q_{B}\left(\lambda_{i}, \lambda_{i}\right) Q_{C}\left(\lambda_{i}, \lambda_{j}\right)\left(\lambda_{i}-\lambda_{j}\right) e_{j}\left(\lambda_{i}\right) e_{i} \\
& +\sum_{m=0}^{k}\left[\left(B\left(\lambda_{i}\right)-B\left(\lambda_{j}\right)\right) e_{i}\left(c_{m}\right) \lambda_{j}^{m} e_{j}\right. \\
& \left.+\left(C\left(\lambda_{i}\right)-C\left(\lambda_{j}\right)\right) e_{j}\left(b_{m}\right) \lambda_{i}^{m} e_{i}\right] .
\end{aligned}
$$

Это выражение эквивалентно равенству

$$
\begin{gathered}
N_{B, C}\left(e_{i}, e_{j}\right)=Q_{B}\left(A, \lambda_{i}\right) Q_{C}\left(A, \lambda_{j}\right) N_{A}\left(e_{i}, e_{j}\right)+\sum_{m=0}^{k}\left[B(A) e_{i}\left(c_{m}\right) A^{m} e_{j}\right. \\
\left.-C(A) e_{j}\left(b_{m}\right) A^{m} e_{i}-e_{i}\left(c_{m}\right) B(A) A^{m} e_{j}+e_{j}\left(b_{m}\right) C(A) A^{m} e_{i}\right]
\end{gathered}
$$

где мы используем соотношение Нийенхейса [1]

$$
N_{A}\left(e_{i}, e_{j}\right)=\left(A-\lambda_{i}\right)\left(A-\lambda_{j}\right)\left[e_{i}, e_{j}\right]+\left(\lambda_{i}-\lambda_{j}\right)\left(e_{i}\left(\lambda_{j}\right) e_{j}+e_{j}\left(\lambda_{i}\right) e_{i}\right) .
$$

Вследствие (2.4) первый член в (3.6) имеет вид

$$
Q_{B}\left(A, \lambda_{i}\right) Q_{C}\left(A, \lambda_{j}\right) N_{A}\left(e_{i}, e_{j}\right)=T_{Q_{B}(z, \lambda) Q_{C}(z, \mu)} N_{A}\left(e_{i}, e_{j}\right) .
$$

Подставляя это выражение в (3.6), мы получаем

$$
\begin{array}{r}
N_{B, C}(u, v)=T_{Q_{B}(z, \lambda) Q_{C}(z, \mu)} N_{A}(u, v)+\sum_{m=0}^{k}\left[B(A) u\left(c_{m}\right) A^{m} v\right. \\
\left.-C(A) v\left(b_{m}\right) A^{m} u-u\left(c_{m}\right) B(A) A^{m} v+v\left(b_{m}\right) C(A) A^{m} u\right],
\end{array}
$$

где $u=e_{i}$ и $v=e_{j}$. Отсюда по билинейности следует формула (3.7) для общих векторов $u$ и $v$. Формула (3.3) совпадает с (3.7) в силу определений (2.2) и (2.12). Поэтому формула $(3.3)$ доказана для любого $(1,1)$-тензора $A_{j}^{i}(x)$ с неравными собственными числами. Применяя замечание 2.1 , мы получаем, что формула (3.3) справедлива для любого $(1,1)$-тензора $A_{j}^{i}(x)$.

СлЕДСТвИЕ 3.1. Для произвольного многочлена $B(A, x)$ тензор Нийенхейса $N_{B, A}(u, v)$ удовлетворяет тождеству

$$
\begin{aligned}
N_{B, A}(u, v)= & \sum_{m=1}^{k} b_{m} \sum_{p+q=m-1} A^{p} N_{A}\left(A^{q} u, v\right) \\
& +\sum_{m=0}^{k}\left[v\left(b_{m}\right) A^{m+1} u-A v\left(b_{m}\right) A^{m} u\right] .
\end{aligned}
$$

Действительно, формула (3.3) для $C(A)=A$ сводится к формуле $(3.8)$, поскольку $c_{1}=1$ и $c_{l}=0$ для $l \neq 1$. 
СлеДСТВИЕ 3.2. Для любого многочлена $B(A, x)$ из (3.1) тензор Нийенхейса $N_{B}(u, v)$ связан с тензором Нийенхейса $N_{A}(u, v)$ формулой

$$
\begin{aligned}
N_{B}(u, v)= & \sum_{m, l=1}^{k} b_{m} b_{l} \sum_{p<m, q<l} A^{m+l-p-q-2} N_{A}\left(A^{p} u, A^{q} v\right) \\
& +\sum_{m=0}^{k}\left[B(A) u\left(b_{m}\right) A^{m} v-B(A) v\left(b_{m}\right) A^{m} u\right. \\
& \left.-u\left(b_{m}\right) B(A) A^{m} v+v\left(b_{m}\right) B(A) A^{m} u\right] .
\end{aligned}
$$

Действительно, по определению (1.1) тензор Нийенхейса $N_{B}(u, v)$ равен тензоpy $N_{B, B}(u, v)(2.6)$. Подставляя $C(A)=B(A)$ в (3.3), мы получаем формулу $(3.9)$.

ЗАмЕчАниЕ 3.1. Эквивалентная форма тождества (3.9) следует из (3.7) после подстановки $C(A)=B(A)$ :

$$
\begin{gathered}
N_{B}(u, v)=T_{Q_{B}(z, \lambda) Q_{B}(z, \mu)} N_{A}(u, v)+\sum_{m=0}^{k}\left[B(A) u\left(b_{m}\right) A^{m} v\right. \\
\left.-B(A) v\left(b_{m}\right) A^{m} u-u\left(b_{m}\right) B(A) A^{m} v+v\left(b_{m}\right) B(A) A^{m} u\right] .
\end{gathered}
$$

Формулы (3.9) и (3.10) дают полное решение задачи Нийенхейса [1], [2] о взаимосвязях между тензорами $N_{B(A)}(u, v)$ и $N_{A}(u, v)$. Для любого многочлена $B(A)$ (3.1) с постоянными коэффициентами формула (3.10) принимает простой вид

$$
N_{B}(u, v)=T_{Q_{B}(z, \lambda) Q_{B}(z, \mu)} N_{A}(u, v) .
$$

СлЕДСТВИЕ 3.3. Для произвольного многочлена $B(A, x)$ тензор Хаантжеса $H_{B}(u, v)$ связан с тензором Хаантжеса $H_{A}(u, v)$ посредством формуль

$$
H_{B}(u, v)=T_{Q_{B}^{2}(z, \lambda) Q_{B}^{2}(z, \mu)} H_{A}(u, v) .
$$

ДоказАТЕльство. Тензор Хаантжеса $H_{B}(u, v)$ связан с тензором Нийенхейса $N_{B}(u, v)$ соотношением

$$
H_{B}(u, v)=B^{2} N_{B}(u, v)+N_{B}(B u, B v)-B\left(N_{B}(B u, v)+N_{B}(u, B v)\right),
$$

которое эквивалентно выражению

$$
H_{B}(u, v)=T_{(B(z)-B(\lambda))(B(z)-B(\mu))} N_{B}(u, v) .
$$

Используя здесь тождество (3.4), мы получаем

$$
H_{B}(u, v)=T_{Q_{B}(z, \lambda) Q_{B}(z, \mu)} T_{D} N_{B}(u, v),
$$

где $D=(z-\lambda)(z-\mu)$. Формула (3.11) следует из (3.12) после подстановки соотношения (3.10) и использования уравнений $(2.8)$ и (2.10). 
СлеДСТвИЕ 3.4. Справедливы следующие тождества, содержащие произвольнье функции $f(x)$ и $g(x)$ : для тензора Нийенхейса

$$
\begin{aligned}
& N_{f(x) A+g(x)}(u, v)=f^{2}(x) N_{A}(u, v) \\
& \quad+f\left[A u(f) A v-A v(f) A u-u(f) A^{2} v+v(f) A^{2} u\right] \\
& \quad+f[A u(g) v-A v(g) u-u(g) A v+v(g) A u]
\end{aligned}
$$

и для тензора Хаантжеса

$$
H_{f(x) A+g(x)}(u, v)=f^{4}(x) H_{A}(u, v) .
$$

Действительно, для оператора

$$
B(A, x)=f(x) A(x)+g(x)
$$

мы имеем

$$
Q_{B}(z, \lambda)=f(x), \quad b_{0}(x)=g(x), \quad b_{1}(x)=f(x) .
$$

Формулы (3.13) и (3.14) следуют из (3.9) и (3.11) после подстановки соотношений (3.16).

ЗАмечание 3.2. Формула (3.14) означает, что тензор Хаантжеса $H_{A}(u, v)$ является калибровочным инвариантом по отношению к калибровочным преобразованиям (3.15). Из формулы (3.13) следует, что тензор Нийенхейса $N_{A}(u, v)$ не является калибровочным инвариантом.

\section{§4. Общие алгебраические тождества для тензоров Нийенхейса и Хаантжеса}

4.1. Для произвольного многочлена $B(A, x)$ (см. (3.1)) мы введем следующий альтернирующий $(1,2)$-тензор:

$$
\begin{aligned}
Z_{B}(u, v)= & \sum_{m=2}^{k} b_{m}(x) \sum_{p+q+r=m-2} A^{p} N_{A}\left(A^{q} u, A^{r} v\right) \\
& +\sum_{m=0}^{k}\left[v\left(b_{m}\right) A^{m} u-u\left(b_{m}\right) A^{m} v\right] .
\end{aligned}
$$

ЛЕмма 4.1. Справедливо тензорное тождество

$$
N_{B, A}(u, v)+N_{B, A}(v, u)=Z_{B}(A u, v)-Z_{B}(u, A v) .
$$

Действительно, это тождество проверяется прямой подстановкой в (4.2) формул (3.8) и (4.1).

ЛЕмма 4.2. Если $(1,1)$-тензор $A_{j}^{i}(x)$ удовлетворяет алгебраическому уравнению $B(A, x)=0$, то справедливо тензорное равенство

$$
Z_{B}(A u, v)=Z_{B}(u, A v) .
$$

Действительно, уравнение $B(A, x)=0$ влечет $N_{B, A}(u, v)=0$. Поэтому уравнение (4.3) следует из тождества (4.2). 
Теорема 4.1. Тензор Нийенхейса $N_{A}(u, v)$ удовлетворяет тензорному тождеству

$$
\sum_{m=2}^{n} a_{m}(x) \sum_{p+q+r=m-2} A^{p} N_{A}\left(A^{q} u, A^{r} v\right)=\sum_{m=0}^{n}\left(u\left(a_{m}\right) A^{m} v-v\left(a_{m}\right) A^{m} u\right),
$$

где $a_{m}(x)$ - коэффичиенты характеристического многочлена

$$
P(\lambda, x)=\operatorname{det}(A(x)-\lambda)=\sum_{m=0}^{n} a_{m}(x) \lambda^{m} .
$$

ДоКАЗАТЕЛЬСТво. Отметим, что уравнение (4.4) имеет вид $Z_{P}(u, v)=0$, где тензор $Z_{P}(u, v)$ определен формулой (4.1) при $B(\lambda, x)=P(\lambda, x)$. Предположим сначала, что $(1,1)$-тензор $A_{j}^{i}(x)$ имеет неравные собственные значения $\lambda_{1}(x), \ldots$ $\ldots, \lambda_{n}(x)$ с собственными векторами $e_{1}(x), \ldots, e_{n}(x)$. По теореме Кэли-Гамильтона справедливо уравнение $P(A, x)=0$. Следовательно, лемма 4.2 и уравнение (4.3) дают $\left(\lambda_{i}-\lambda_{j}\right) Z_{P}\left(e_{i}, e_{j}\right)=0$. Отсюда в силу билинейности и кососимметричности тензора $Z_{P}(u, v)$ следует $Z_{P}(u, v)=0$. Используя замечание 2.1 , мы получаем, что уравнение $Z_{P}(u, v)=0$ и эквивалентное тождество (4.4) справедливы для любого $(1,1)$-тензора $A_{j}^{i}(x)$.

СлеДСтвИЕ 4.1. Тензор Хаантжеса $H_{A}(u, v)$ удовлетворяет тензорному тождеству

$$
\sum_{m=2}^{n} a_{m}(x) \sum_{p+q+r=m-2} A^{p} H_{A}\left(A^{q} u, A^{r} v\right)=0,
$$

которое имеет вид

$$
\widetilde{Z}_{P}(u, v)=T_{R_{P}(z, \lambda, \mu)} H_{A}(u, v)=0,
$$

где $P=P(\lambda, x)$ - характеристический многочлен (4.5).

ДоказАТЕЛЬСтво. Применяя оператор $T_{D(z, \lambda, \mu)}$, где $D$ имеет вид $(2.9)$, к тождеству (4.4) и используя соотношения (2.8) и (2.10), мы получаем тождество (4.6). Вследствие формул (2.16) и (2.17) это тождество имеет вид (4.7).

4.2. При $n=2$ характеристический многочлен (4.5) имеет вид

$$
P(\lambda)=\lambda^{2}-(\operatorname{Tr} A) \lambda+\operatorname{det} A=0,
$$

поэтому из тождества (4.4) следует

$$
N(u, v)=u(\operatorname{det} A) v-v(\operatorname{det} A) u-u(\operatorname{Tr} A) A v+v(\operatorname{Tr} A) A u .
$$

Для $n=3$ характеристический многочлен (4.5) есть

$$
P(\lambda)=\lambda^{3}-(\operatorname{Tr} A) \lambda^{2}+a_{1} \lambda-\operatorname{det} A=0,
$$

где $2 a_{1}=(\operatorname{Tr} A)^{2}-\operatorname{Tr}\left(A^{2}\right)$. Поэтому тождество (4.4) влечет равенство

$$
\begin{gathered}
(A-\operatorname{Tr} A) N_{A}(u, v)+N_{A}(A u, v)+N_{A}(u, A v)=v(\operatorname{det} A) u-u(\operatorname{det} A) v \\
-v\left(a_{1}\right) A u+u\left(a_{1}\right) A v+v(\operatorname{Tr} A) A^{2} v-u(\operatorname{Tr} A) A^{2} u .
\end{gathered}
$$


При $n=3$ тождество (4.6) принимает вид

$$
(A-\operatorname{Tr} A) H_{A}(u, v)+H_{A}(A u, v)+H_{A}(u, A v)=0 .
$$

Следствием тождества (4.8) является важное свойство, что тензор Хаантжеса определяет структуру алгебр Ли в касательных пространствах $T_{x}\left(M^{3}\right)$ (см. далее $\S 5)$.

\section{§5. Специальные алгебраические тождества для $n=3$}

Обозначим

$$
N(u, v)=N_{A}(u, v), \quad H(u, v)=H_{A}(u, v), \quad M(u, v)=N(A u, v)-A N(u, v)
$$

и введем линейные операторы $N_{u}(v)=N(u, v), H_{u}(v)=H(u, v), M_{u}(v)=$ $M(u, v)$. Очевидно, мы имеем

$$
M_{u}=N_{A u}-A N_{u}, \quad H_{u}=\left[M_{u}, A\right] .
$$

Уравнения (5.1) влекут равенства

$$
\operatorname{Tr} H_{u}=0, \quad \operatorname{Tr}\left(A^{k} H_{u}\right)=0, \quad k=1,2, \ldots .
$$

Рассмотрим симметрическую билинейную форму $(u, v)_{H}=\operatorname{Tr}\left(H_{u} H_{v}\right)$ и альтернирующий $(1,3)$-тензор [4]-[6]

$$
B_{H}(u, v, w)=H(H(u, v), w)+H(H(v, w), u)+H(H(w, u), v) .
$$

Отсюда мы получаем для линейного оператора $B_{u v}(w)=B_{H}(u, v, w)$, что

$$
B_{u v}=H_{H(u, v)}-\left[H_{u}, H_{v}\right] .
$$

Теорема 5.1. При $n=3$ тензоры Нийенхейса и Хаантжсеса имеют следующие свойства:

1) тензор Хаантжеса $H(u, v)$ определяет структуру алгебр Ли в касательных пространствах $T_{x}\left(M^{3}\right)$;

2) операторы $H_{w}$ являются кососимметрическими по отношению к билинейной форме $(u, v)_{H}$ :

$$
\left(H_{w} u, v\right)_{H}=-\left(u, H_{w} v\right)_{H}
$$

3) операторы $A_{j}^{i}(x)$ являются симметрическими по отношению $к$ форме $(u, v)_{H}$ :

$$
(A u, v)_{H}=(u, A v)_{H}
$$

4) тензор Нийенхейса $N(u, v)$ удовлетворяет следующему тождеству:

$$
\begin{gathered}
(B(A) N(u, v), w)_{H}+(B(A) N(v, w), u)_{H}+(B(A) N(w, u), v)_{H} \\
=R_{B}\left(\lambda_{1}, \lambda_{2}, \lambda_{3}\right)(H(u, v), w)_{H},
\end{gathered}
$$

где $B(A, x)$ - произвольный многочлен $(3.1), R_{B}\left(\lambda_{1}, \lambda_{2}, \lambda_{3}\right)$ - соответствуюший симметрический многочлен (2.14), $\lambda_{1}(x), \lambda_{2}(x)$ и $\lambda_{3}(x)$ - собственные значения (1,1)-тензора $A_{j}^{i}(x) u u, v, w \in T_{x}\left(M^{3}\right)$ - произвольные касательнье векторы. 
ДокАЗАТЕЛьство. 1) При $n=3$ альтернирующий тензор $B_{i j k}^{s}$ имеет ненулевые компоненты только при $i \neq j \neq k$. Пусть $s=k$; тогда мы имеем

$$
B_{i j k}^{k}=B_{i j k}^{k}+B_{i j i}^{i}+B_{i j j}^{j}=\operatorname{Tr}\left(B_{e_{i} e_{j}}\right) .
$$

Уравнения (5.2) и (5.4) дают $\operatorname{Tr}\left(B_{u v}\right)=0$. Следовательно, все компоненты $B_{i j k}^{s}$ равны нулю или тензор $B_{H}(u, v, w)$ тождественно равен нулю.

2) Уравнение (5.5) следует из общей теории алгебр Ли, поскольку при $n=3$ билинейная форма $(u, v)_{H}$ является формой Картана-Киллинга.

3) Предположим сначала, что оператор $A_{j}^{i}(x)$ имеет не равные (комплексные) собственные значения $\lambda_{1}(x), \lambda_{2}(x), \lambda_{3}(x)$ и соответствуюшие собственные векторы $e_{1}(x), e_{2}(x), e_{3}(x), A e_{i}=\lambda_{i} e_{i}$, принадлежащие комплексификации касательного расслоения $T\left(M^{3}\right)$.

Из формулы (2.7) имеем

$$
H\left(e_{i}, e_{j}\right)=\left(A-\lambda_{i}\right)\left(A-\lambda_{j}\right) N\left(e_{i}, e_{j}\right) .
$$

Применяя оператор $\left(A-\lambda_{k}\right)$ к $(5.8)$ и используя теорему Кэли-Гамильтона при $n=3$, получаем

$$
\left(A-\lambda_{k}\right) H\left(e_{i}, e_{j}\right)=P(A) N\left(e_{i}, e_{j}\right)=0 .
$$

Отсюда мы находим $H\left(e_{i}, e_{j}\right)=H_{i j}^{k} e_{k}$ при $i \neq j \neq k$. Эти соотношения влекут $H_{e_{i}} H_{e_{j}} e_{i}=H_{j i}^{k} H_{i k}^{j} e_{j}, H_{e_{i}} H_{e_{j}} e_{j}=0, H_{e_{i}} H_{e_{j}} e_{k}=0$. Следовательно,

$$
\left(e_{i}, e_{j}\right)_{H}=\operatorname{Tr}\left(H_{e_{i}} H_{e_{j}}\right)=0, \quad i \neq j .
$$

Уравнение (5.6) является очевидным следствием уравнений $A e_{i}=\lambda_{i} e_{i}$ и (5.9). Используя замечание 2.1, мы получаем, что тождество (5.6) справедливо для произвольного $(1,1)$-тензора $A_{j}^{i}(x)$.

4) Предположим сначала, что тензор $A_{j}^{i}(x)$ имеет различные собственные значения $\lambda_{k}(x)$. Левая часть равенства (5.7) является альтернирующей, поскольку тензор Нийенхейса является альтернирующим. Правая часть (5.7) является альтернирующей в силу тождества (5.5). Поэтому достаточно доказать тождество (5.7) только для собственных векторов $u=e_{1}(x), v=e_{2}(x), w=e_{3}(x)$. Применим оператор $B(A, x)$ (см. (3.1)) к равенству (5.8) и затем рассмотрим скалярное произведение $(u, v)_{H}$ результирующего вектора с собственным вектором $e_{k}$. Используя тождество (5.6) и уравнения $A e_{i}=\lambda_{i} e_{i}$, получаем равенство

$$
\left(B(A) N\left(e_{i}, e_{j}\right), e_{k}\right)_{H}=B\left(\lambda_{k}\right)\left(\lambda_{k}-\lambda_{i}\right)^{-1}\left(\lambda_{k}-\lambda_{j}\right)^{-1}\left(H\left(e_{i}, e_{j}\right), e_{k}\right)_{H} .
$$

Вследствие (5.5) находим

$$
\begin{aligned}
& \left(B(A) N\left(e_{i}, e_{j}\right), e_{k}\right)_{H}+\left(B(A) N\left(e_{j}, e_{k}\right), e_{i}\right)_{H}+\left(B(A) N\left(e_{k}, e_{i}\right), e_{j}\right)_{H} \\
& \quad=R_{B}\left(\lambda_{i}, \lambda_{j}, \lambda_{k}\right)\left(H\left(e_{i}, e_{j}\right), e_{k}\right)_{H},
\end{aligned}
$$

где

$$
\begin{aligned}
R_{B}\left(\lambda_{i}, \lambda_{j}, \lambda_{k}\right)= & B\left(\lambda_{i}\right)\left(\lambda_{i}-\lambda_{k}\right)^{-1}\left(\lambda_{i}-\lambda_{j}\right)^{-1}+B\left(\lambda_{j}\right)\left(\lambda_{j}-\lambda_{i}\right)^{-1}\left(\lambda_{j}-\lambda_{k}\right)^{-1} \\
& +B\left(\lambda_{k}\right)\left(\lambda_{k}-\lambda_{j}\right)^{-1}\left(\lambda_{k}-\lambda_{i}\right)^{-1}
\end{aligned}
$$


Из тождества (2.15) следует, что функция (5.10) совпадает с симметрическим многочленом $R_{B}(z, \lambda, \mu)(2.14)$. Поэтому тождество (5.7) доказано в силу билинейности, если $\lambda_{1}(x) \neq \lambda_{2}(x) \neq \lambda_{3}(x)$. Применяя замечание 2.1 , мы получаем, что тождество (5.7) справедливо для произвольного $(1,1)$-тензора $A_{j}^{i}(x)$.

ЗАмЕчАниЕ 5.1. Для любого многочлена второй степени

$$
B(A, x)=b_{2}(x) A^{2}(x)+b_{1}(x) A(x)+b_{0}(x)
$$

из формулы $(2.14)$ имеем $R_{B}(z, \lambda, \mu)=b_{2}(x)$. Поэтому тождество (5.7) при $B(A)=1, A, A^{2}$ влечет равенства

$$
\begin{gathered}
(N(u, v), w)_{H}+(N(v, w), u)_{H}+(N(w, u), v)_{H}=0 \\
(A N(u, v), w)_{H}+(A N(v, w), u)_{H}+(A N(w, u), v)_{H}=0 \\
\left(A^{2} N(u, v), w\right)_{H}+\left(A^{2} N(v, w), u\right)_{H}+\left(A^{2} N(w, u), v\right)_{H}=(H(u, v), w)_{H} .
\end{gathered}
$$

Эти тождества имеют обобщения (см. [5], [6]) на $n$-мерньй случай для специальных $(1,1)$-тензоров $A_{j}^{i}(x)$, соответствуюших гамильтоновым системам (1.2).

\section{Список литературы}

1. Nijenhuis A. $X_{n-1}$-forming sets of eigenvectors // Proc. Kon. Ned. Akad. Amsterdam. 1951. V. 54. P. 200-212.

2. Nijenhuis A. Jacobi-type identities for bilinear differential concomitants of certain tensor fields // Proc. Kon. Ned. Akad. Amsterdam. 1955. V. 58. P. 390-403.

3. Haantjes J. On $X_{m}$-forming sets of eigenvectors // Proc. Kon. Ned. Akad. Amsterdam. 1955. V. 58. P. $158-162$.

4. Bogoyavlenskij O. I. Existence of Riemann invariants and Hamiltonian structures // C.R. Math. Rep. Acad. Sci. Canada. 1993. V. 15. P. 143-148.

5. Bogoyavlenskij O.I. Necessary conditions for existence of non-degenerate Hamiltonian structures // Commun. Math. Phys. 1996. V. 182. P. 253-290.

6. Bogoyavlenskij O. I. Courant's problems and their extensions // Proceedings of VII International Conference on hyperbolic problems, International Series of Numerical Mathematics. V. 129. Basel: Birkhauser Verlag, 1999. P. 97-104.

7. Lichnerowicz A. Les varietes de Poisson et leurs algebres de Lie associees // J. Diff. Geom. 1977. V. 12. P. 253-300.

8. Nijenhuis A., Richardson R. W. Deformations of Lie algebra structures // J. Math. Mech. 1967. V. 17. P. 89-105.

9. Newlander A., Nirenberg L. Complex analytic coordinates in almost-complex manifolds // Ann. Math. 1957. V. 65. P. 391-404.

10. DeBarros C. M. Varietes presque hor-complexes // C.R. Acad. Sc. Paris. 1965. V. 260. P. $1543-1546$.

11. Lehmann-Lejeune J. Etude des formes differentielles liee a certaines $G$-structures // C.R. Acad. Sc. Paris. 1965. V. 260. P. 1838-1841.

12. Lavandier $J$. Tenseur de Nijenhuis et integralite des $G$-structures definies par une 1-forme vectorielle, $O$-deformable // C. R. Acad. Sc. Paris. 1994. V. 318. P. 135-138.

13. Гельфанд И. М., Дорфман И. Я. Скобка Схоутена и гамильтоновы операторы // Функц. анализ и его прилож. 1980. Т. 14. №3. С. 71-74.

14. Magri F., Morosi C. A geometrical characterization of integrable Hamiltonian systems through the theory of the Poisson-Nijenhuis manifolds // Quaderno S/19. Preprint / Universita di Milano, 1984.

15. Magri F. A simple model of an integrable Hamiltonian system // J.Math. Phys. 1978. V. 19. P. $1156-1162$. 
16. Kosmann-Schwarzbach Y., Magri F. Poisson-Nijenhuis structures // Ann. Inst. Henri Poincare. 1990. V. 53. P. 35-81.

17. Magri F., Morosi C., Ragnisco O. Reduction techniques for infinite-dimensional Hamiltonian systems: some ideas and applications // Commun. Math. Phys. 1985. V. 99. P. $115-140$.

18. Magri F., Morosi C., Tondo G. Nijenhuis $G$-manifold and Lenard bicomplexes: a new approach to $K P$ systems // Commun. Math. Phys. 1988. V. 115. P. 457-475.

19. Stone A.P. Generalized conservation laws // Proceed. Amer. Math. Soc. 1967. V. 18. P. 868-873.

20. Stone A. P. Some remarks on the Nijenhuis tensor // Can. J. Math. 1973. V. 25. P. 903-907.

21. Дубровин Б.А., Новиков С. П. Гидродинамика слабо деформированных солитонных решеток. Дифференциальная геометрия и гамильтонова теория // УМН. 1989. Т. 44. №6. C. 29-98.

22. Bogoyavlenskij O.I. Necessary conditions for the existence of conservation laws for systems of partial differential equations // Symposia Gaussiana Conf. Berlin-N.Y.: Walter de Gruyter Co., 1995. P. 359-375.

Математический институт им. В.А. Стеклова РАН,

Поступило в редакцию

Квинский университет, Кингстон, Канада

25.05.2004

E-mail: bogoyavl@mast.queensu.ca 